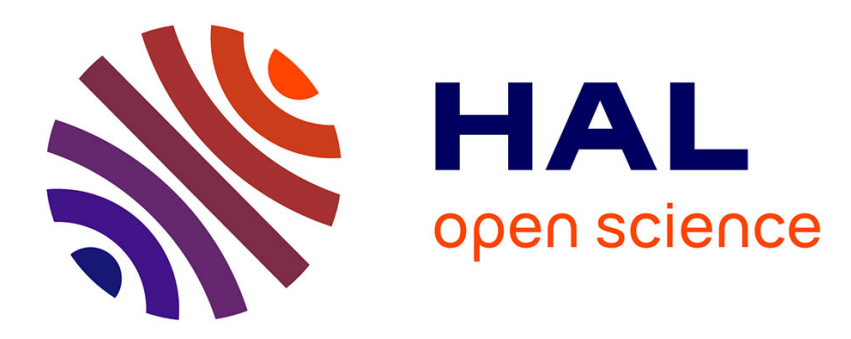

\title{
Non-Poisson statistics of settling spheres
}

Laurence Bergougnoux, Elisabeth Guazzelli

\section{To cite this version:}

Laurence Bergougnoux, Elisabeth Guazzelli. Non-Poisson statistics of settling spheres. Physics of Fluids, 2009, 21, pp.091701. 10.1063/1.3231828 . hal-01066993

\section{HAL Id: hal-01066993 https://hal.science/hal-01066993}

Submitted on 22 Sep 2014

HAL is a multi-disciplinary open access archive for the deposit and dissemination of scientific research documents, whether they are published or not. The documents may come from teaching and research institutions in France or abroad, or from public or private research centers.
L'archive ouverte pluridisciplinaire HAL, est destinée au dépôt et à la diffusion de documents scientifiques de niveau recherche, publiés ou non, émanant des établissements d'enseignement et de recherche français ou étrangers, des laboratoires publics ou privés. 


\title{
Non-Poisson statistics of settling spheres
}

\author{
Laurence Bergougnoux and Élisabeth Guazzelli \\ IUSTI CNRS UMR 6595, Polytech'Marseille, Aix-Marseille Université (U1), 5 rue Enrico Fermi, \\ 13453 Marseille Cedex 13, France
}

(Received 8 June 2009; accepted 12 August 2009; published online 22 September 2009)

\begin{abstract}
Direct tracking of the particle positions in a sedimenting suspension indicates that the particles are not simply randomly distributed. The initial mixing of the suspension leads to a microstructure which consists of regions devoid of particles surrounded by regions where particles have an excess of close neighbors and which is maintained during sedimentation. (C) 2009 American Institute of
\end{abstract} Physics. [doi:10.1063/1.3231828]

The settling of a collection of non-Brownian particles in a viscous fluid is a basic problem in suspension dynamics but is remarkably difficult to tackle. The hydrodynamic interactions between the particles are long ranged and of manybody character leading to a complex dynamical behavior. In the absence of Brownian motion providing a randomizing mechanism, the suspension microstructure is determined by the hydrodynamics which in turn depends on the microstructure, a feature that makes sedimentation a particularly challenging problem relevant to nonequilibrium statistical physics.

In the absence of inertia, a single sphere settles at the Stokes velocity $V_{S}=(2 / 9) a^{2}\left(\rho_{p}-\rho_{f}\right) g / \mu$, where $a$ is the sphere radius, $\rho_{p}$ is the density of the particles, $\rho_{f}$ that of the fluid, and $\mu$ of the fluid viscosity. The average sedimentation speed of an ensemble of settling spheres decreases rapidly with increasing particle volume fraction $\phi$ due to the fluid backflow induced by the particles falling down to the impenetrable bottom of the container. The first order correction of $-6.55 \phi$ to the Stokes velocity was computed by Batchelor ${ }^{1}$ assuming low $\phi$ and randomly dispersed spheres. Following Batchelor's assumption of a dilute random distribution of sedimenting spheres, Caflisch and $\mathrm{Luke}^{2}$ and $\mathrm{Hinch}^{3}$ predicted that velocity fluctuations (standard deviation) should diverge with the size of the container. Experiments in large containers $^{4-6}$ show indeed that fluctuations are of the order of the mean in magnitude and of the order of the cell width in size just after the initial mixing of the suspension. But, these large-scale fluctuations are transient and give place to smaller-scale fluctuations of smaller magnitude and size $\approx 20 a \phi^{-1 / 3}$, which remain constant (in a plateau region) until the arrival of the upper sedimentation front between the suspension and the clear fluid. ${ }^{4,7}$

This screening of the fluctuations stimulated numerous theoretical models and simulations. ${ }^{8-14}$ Koch and Shaqfeh ${ }^{8}$ proposed a Debye-like screening which assumed a specific particle distribution induced by three-particle interactions (more precisely a net average deficit of one particle in the vicinity of any other particles). Several other bulk mechanisms leading to nonuniform microstructures have been proposed including a stochastic convection-diffusion model ${ }^{10}$ and analogy with high Prandtl number turbulence. ${ }^{11}$ However, screening has not been observed in numerical simula- tions of homogeneous suspension with periodic boundary conditions. ${ }^{9}$ It is only the inclusion of bottom and top boundaries acting as sink of the large-scale disturbances that leads to damping and subsequent saturation of the velocity fluctuations, ${ }^{12,14}$ similar to what is seen in experiments ${ }^{4-6}$ and in agreement with an earlier suggestion of Hinch. ${ }^{3}$ Simulations with bounding walls ${ }^{14}$ show that monodisperse suspensions develop a strongly anisotropic long-range microstructure during the settling process with vanishing density fluctuations in the horizontal plane. Conversely, in polydisperse suspensions, the microstructure appears to be randomized by the varying settling speeds, but the particle velocity fluctuations are seen to be damped by stratification due to differential settling ${ }^{14}$ rather than interface diffusion. ${ }^{13}$ In contrast, in experiments, the reduction in the velocity fluctuations does not seem to be fully controlled by local stratification as same evolution of the velocity fluctuations is seen despite different polydispersities or different concentration gradients created by the initial mixing. ${ }^{6}$

Obtaining information on the microstructure is important per se and may help in understanding the behavior of the velocity fluctuations. However, it proves to be rather difficult in experiments. While the structure factor can be obtained using light scattering in colloidal suspensions, it is trickier for non-Brownian suspensions because of the large size of the particles compared to the wavelength of light. An analogous scattering technique for noncolloidal suspensions based on pulsed field gradient nuclear magnetic resonance has been proposed instead. The application of the technique to sedi-

TABLE I. Particle characteristics: mean radius $\langle a\rangle$ and standard deviation $\sigma_{a}$, sphere density $\rho_{p}$, Stokes velocity $V_{S}$, and sphere Reynolds number $\operatorname{Re}_{p}=V_{S}\langle a\rangle \rho_{f} / \mu$.

\begin{tabular}{lccccc}
\hline \hline Batch & $\begin{array}{c}\langle a\rangle \\
(\mathrm{cm})\end{array}$ & $\begin{array}{c}\sigma_{a} /\langle a\rangle \\
(\%)\end{array}$ & $\begin{array}{c}\rho_{p} \\
\left(\mathrm{~g} / \mathrm{cm}^{3}\right)\end{array}$ & $\begin{array}{c}V_{S} \\
(\mathrm{~cm} / \mathrm{s})\end{array}$ & $\begin{array}{c}\mathrm{Re}_{p} \\
\left(10^{-5}\right)\end{array}$ \\
\hline A & 0.0202 & 2.5 & $2.50 \pm 0.04$ & $0.0136 \pm 0.0009$ & 2.7 \\
B & 0.0152 & 2.5 & $4.11 \pm 0.07$ & $0.0158 \pm 0.0010$ & 2.3 \\
$\mathrm{C}$ & 0.0148 & 5.6 & $4.11 \pm 0.07$ & $0.0150 \pm 0.0018$ & 2.1 \\
$\mathrm{D}$ & 0.0187 & 11.3 & $2.50 \pm 0.04$ & $0.0117 \pm 0.0027$ & 2.1 \\
\hline \hline
\end{tabular}



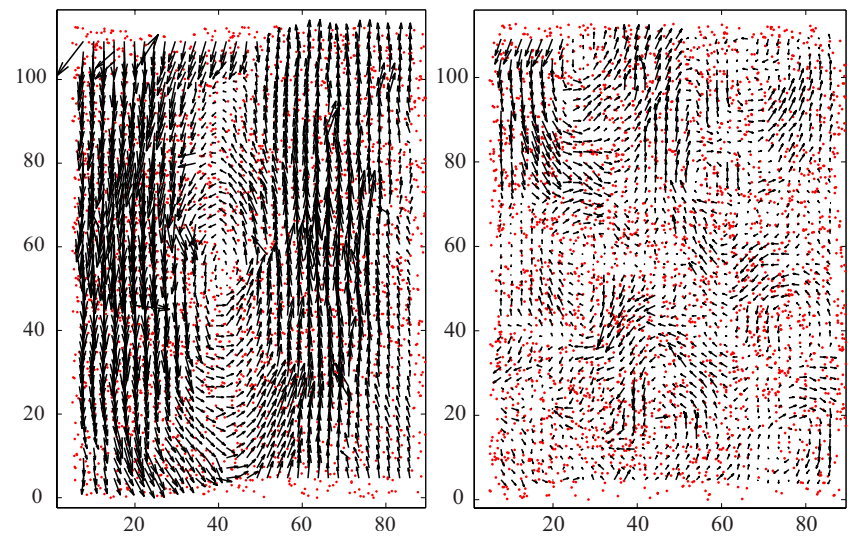

FIG. 1. (Color online) Particle velocity-fluctuation field and center-of-mass positions [gray (red online)] for $t / t_{s}=0$ (left) and $t / t_{s}=729$ (right) for particles of batch B at $\phi=0.3 \%$. Distance is plotted in mean interparticle spacing, $\langle a\rangle \phi^{-1 / 3}$.

mentation has shown that the pair distribution function presents an excess of close particles on a range of about one particle diameter. ${ }^{15}$ It is also possible to track the positions of spheres using imaging techniques and directly infer information about the microstructure. Lei et al. ${ }^{16}$ studied the fluctuations in number density within sampling windows in central region of their experimental cell. Initially selected random fluctuations (Poisson statistics) have been found to become suppressed with increasing test volume dimension. Several questions are nonetheless left open by these two results available in the literature. One may indeed wonder whether the initial mixing leads to a random positioning of the particles or to an excess (or deficit) of close neighbors and how this initial positioning (yet to be determined) evolves during the sedimentation process. To tackle these questions, we have measured the number density statistics and its evolution in a sedimenting suspension by a method similar to that of Lei et al. ${ }^{16}$

Four batches of glass spheres having different polydispersities were used in the experiments, see Table I. The fluid used was silicone oil 47V1000, which had a viscosity $\mu=10.0 \pm 0.3 \mathrm{P}$ and a density $\rho_{f}=0.965 \pm 0.007 \mathrm{~g} \mathrm{~cm}^{-3}$ at the air-conditioned room temperature of $25 \pm 1{ }^{\circ} \mathrm{C}$. The sedimentation experiments were performed in glass-wall vessels of different cross section $(20 \times 20,10 \times 10$, and 4 $\times 4 \mathrm{~cm}^{2}$ for particles of batch $\mathrm{C}$ and $10 \times 10 \mathrm{~cm}^{2}$ for particles of batches $\mathrm{A}, \mathrm{B}$, and D) filled with a fluid height

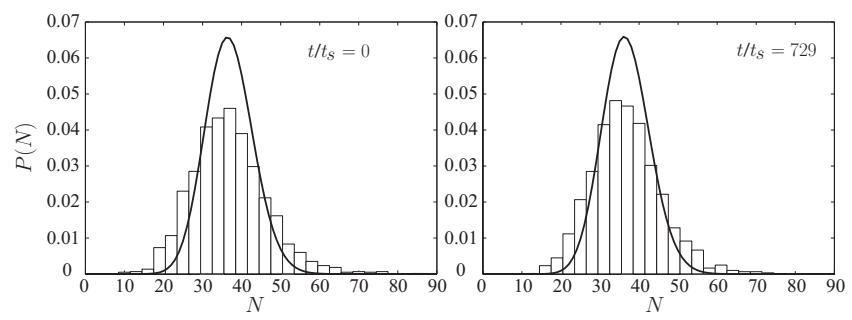

FIG. 2. Particle occupancy distributions for $t / t_{s}=0$ (left) and $t / t_{s}=729$ (right) for particles of batch B at $\phi=0.3 \%$. The solid curves represent the corresponding Poisson distributions.

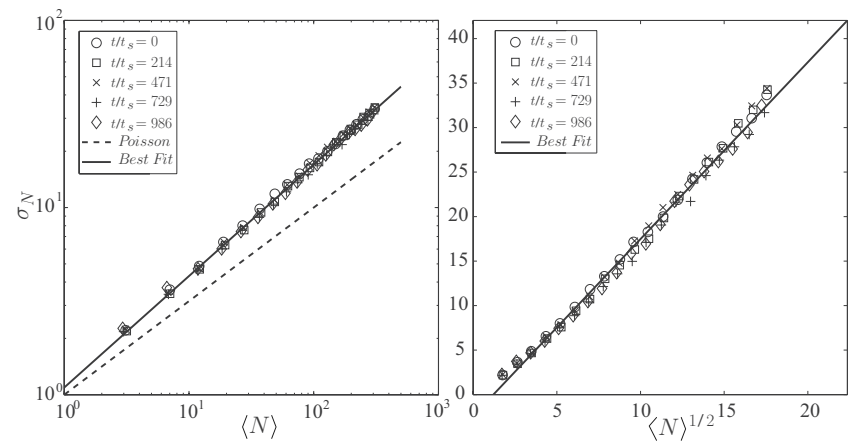

FIG. 3. Standard deviation $\sigma_{N}$ of the number of particles vs $\langle N\rangle$ in $\log -\log$ coordinates (left) and vs $\langle N\rangle^{1 / 2}$ (right) for particles of batch B at $\phi=0.3 \%$. The dotted line in the left graph represents the Poisson law.

$H=40 \mathrm{~cm}$. Most of the experiments were done at the same initial volume fraction $\phi_{0}=0.3 \%$ but a series of experiments with particles of batch $\mathrm{B}$ in the cell having a cross section $10 \times 10 \mathrm{~cm}^{2}$ was also undertaken for volume fractions varying from $0.1 \%$ to $0.8 \%$. Each sedimentation experiment initially consisted of mixing the suspension by moving a small propeller (of size $\approx 2 \mathrm{~cm}$ ) within the suspension for $\approx 10 \mathrm{~min}$ in order to obtain a visually uniform particle distribution throughout the suspension. The mixing process was performed in the same way for the different runs (typically ten), as described previously by Chehata et al. ${ }^{6}$ The principle of this procedure was to turn first the sediment into a concentrated homogeneous suspension in the bottom $5 \mathrm{~cm}$ of the vessel and then to extend the mixing progressively upward until the complete volume of the cell was homogenized. The starting time of each run corresponded to the cessation of mixing.

In the course of measuring particle velocities using particle image velocimetry, we captured images of particles illuminated by a light sheet (of thickness $\approx 1 \mathrm{~mm}$ ) produced by two $15 \mathrm{~mW}$ laser diodes facing each other. The imaging window was typically of height $10 \mathrm{~cm}(\approx H / 4)$ placed $25.5 \mathrm{~cm}$ below the liquid-air interface and sampled the entire
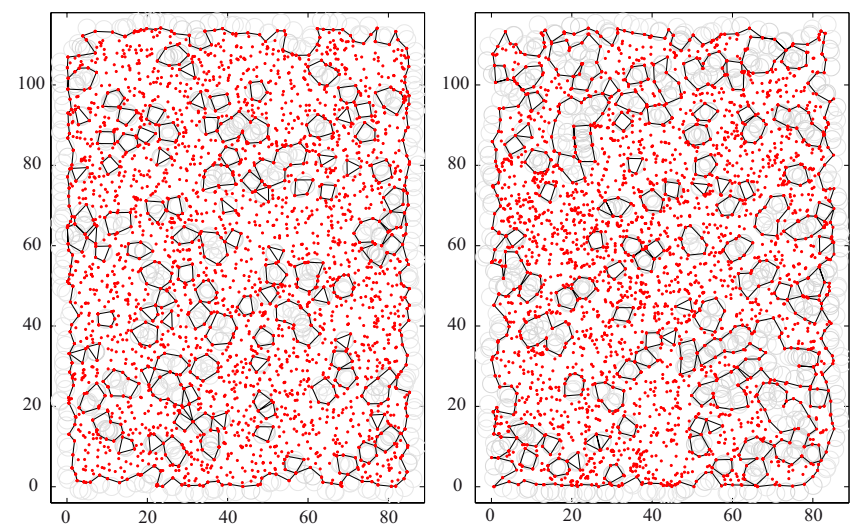

FIG. 4. (Color online) Particle center-of-mass positions [gray (red online)] and holes found by $\alpha$-shapes for fake random positioning (left) and for experimental data (right) for batch $\mathrm{C}$ with $\phi=0.3 \%$ at $t / t_{s}=0$. Distance is plotted in mean interparticle spacing, $\langle a\rangle \phi^{-1 / 3}$. 
TABLE II. Exponent $n$ and coefficient $c$ vs polydispersity at $\phi=0.3 \%$ in the $10 \times 10 \mathrm{~cm}^{2}$ cell.

\begin{tabular}{lcccc}
\hline \hline Batch & $\mathrm{A}$ & $\mathrm{B}$ & $\mathrm{C}$ & $\mathrm{D}$ \\
$\sigma_{a} /\langle a\rangle(\%)$ & 2.5 & 2.5 & 5.6 & 11.3 \\
\hline$n$ & $0.55 \pm 0.03$ & $0.60 \pm 0.02$ & $0.63 \pm 0.04$ & $0.71 \pm 0.02$ \\
$c$ & $1.68 \pm 0.15$ & $2.03 \pm 0.26$ & $2.37 \pm 0.49$ & $3.25 \pm 0.21$ \\
\hline \hline
\end{tabular}

cell cross section. Pairs of images separated by $\approx$ one Stokes time $=a / V_{S}$ were captured every $40 \mathrm{~s}$ and were processed to obtain a two-dimensional velocity-vector map. 5 The same images were used to study particle occupancy statistics. First, we thresholded the gray-level intensity of the images. Second, these images were made binary and the center of mass of the particles was determined. To deduce precisely the threshold to be used, we estimated the number of particles inside the light sheet and used a threshold value, which gave approximately this number. The error bar on the number of particles in the sheet provided upper and lower bounds of the threshold, which were used to determine uncertainties in the processed data. Third, using the method of Lei et al. ${ }^{16}$ we have determined the particle occupancy distribution by counting the number of particles $N$ within a square box of fixed area, which was randomly positioned in each of the images of the different runs corresponding to the same time.

Figure 1 shows the typical particle velocity-fluctuation field and center-of-mass positions obtained in the measuring window $\left(\approx 10 \times 10 \mathrm{~cm}^{2}\right)$ for a single run just after cessation of mixing, where the velocity fluctuations are $\approx$ cell width (left) and in the fluctuation-velocity plateau, where they are $\approx 20 a \phi^{-1 / 3}$ (right). The corresponding particle occupancy distributions obtained from data analysis of ten runs and for a given sampling box of $\approx 1.2 \times 1.2 \mathrm{~cm}^{2}$ are displayed in Fig. 2. The solid curves indicate the Poisson distribution for the same $\langle N\rangle$. Clearly, the observed distributions are shorter and wider than a Poisson distribution. The standard deviations $\sigma_{N}$ of the number of particles are plotted versus the average number of particles $\langle N\rangle$ for different sampling boxes ranging from $0.34 \times 0.34$ to $3.4 \times 3.4 \mathrm{~cm}^{2}$ and for different times in Fig. 3 (left). Two important observations can be made. First, $\sigma_{N}$ is certainly not $=\langle N\rangle^{1 / 2}$ (Poisson statistics) but is $=\langle N\rangle^{n}$ with an exponent $n>0.5$. Second, there is no evolution of this power law with time until the sedimentation front enters the imaging window. Using all the data from the different times, $\log \left(\sigma_{N}\right)$ can be fitted by a linear function of $\log (\langle N\rangle)$ using the method of weighted least squares and one finds $n=0.6$ in the present case.
TABLE IV. Exponent $n$ and coefficient $c$ vs cell cross section at $\phi=0.3 \%$ for batch $\mathrm{C}$.

\begin{tabular}{lccc}
\hline \hline Section $(\mathrm{cm})$ & 4 & 10 & 20 \\
\hline$n$ & $0.52 \pm 0.03$ & $0.63 \pm 0.04$ & $0.77 \pm 0.02$ \\
$c$ & $1.43 \pm 0.22$ & $2.03 \pm 0.26$ & $4.90 \pm 0.40$ \\
\hline \hline
\end{tabular}

This power-law finding is purely empirical but indicates that the particles are not simply randomly distributed inside the sheet volume. A closer inspection of the particle centerof-mass arrangement using the concept of $\alpha$-shapes ${ }^{17}$ (based on Delaunay triangulation and used for shape reconstruction) shows clearly regions devoid of particles surrounded by regions where particles are in excess, see Fig. 4 (right). This leads to an alternative way of interpreting the data suggested by Hinch. ${ }^{18}$ The key idea is to consider that there are two independently random effects that can be added by squares of variances: fluctuations in particle number coming from randomly positioning the particles (with standard deviation $=N^{1 / 2}$ ) and those coming from randomly positioning the holes (with standard deviation $=\left(V_{h} / V\right) N N_{h}^{1 / 2}$, where $N_{h}$ and $V_{h}$ are, respectively, the hole number and volume and $V$ is the entire volume). This results in a standard deviation still $\propto N^{1 / 2}$ but with a coefficient $c>1$, i.e., $\sigma_{N}=c N^{1 / 2}$ with $c=\left[1+\left(V_{h} / V\right)^{2} N N_{h}\right]^{1 / 2}$. Note that $c$ depends upon the number of particles in holes, $N V_{h} / V$, and the volume fraction of holes, $N_{h} V_{h} / V$. The comparison with this model is given in Fig. 3 (right). The agreement is good (although the correlation coefficient of the linear fit is not as good as in the case of the power law) and holds for the different sedimentation times with $c=2$ in this case. It is important to mention that these two behaviors (power law or Poisson with a coefficient) are representative of all experiments. However, the exponent $n$ and the coefficient $c$ are seen to increase with increasing polydispersity, volume fraction, and cell size, as indicated in Tables II-IV.

To quantify the holes, the experimental data have been processed using $\alpha$-shapes to obtain the hole number $N_{h \text {,exp }}$ and the hole area $A_{h \text {,exp. }}$. Figure 5 (left) shows a typical time evolution of hole number $N_{h \text {,exp }}$ and area $A_{h, \text { exp }}$. The hole number $N_{h, \exp }$ has been normalized by the hole number $N_{h, \text { random }}$ found when randomly positioning point particles, see Fig. 4 (left). It does not vary during the sedimentation process (apart from statistical fluctuations) and is smaller than $N_{h \text {,random }}$ (by an amount of $\approx 20 \%$ ). Similarly, the hole area $A_{h, \exp }$ stays constant at $\approx\left(7\langle a\rangle \phi^{-1 / 3}\right)^{2}$. This absence of

TABLE III. Exponent $n$ and coefficient $c$ vs volume fraction $\phi$ for batch B in the $10 \times 10 \mathrm{~cm}^{2}$ cell.

\begin{tabular}{lcccccc}
\hline $\begin{array}{l}\phi \\
(\%)\end{array}$ & 0.1 & 0.2 & 0.3 & 0.4 & 0.5 & 0.8 \\
\hline$n$ & $0.57 \pm 0.03$ & $0.55 \pm 0.02$ & $0.60 \pm 0.02$ & $0.67 \pm 0.02$ & $0.73 \pm 0.03$ & $0.87 \pm 0.02$ \\
$c$ & $1.56 \pm 0.14$ & $1.42 \pm 0.12$ & $2.03 \pm 0.26$ & $2.57 \pm 0.23$ & $3.68 \pm 0.49$ & $10.07 \pm 0.96$ \\
\hline \hline
\end{tabular}




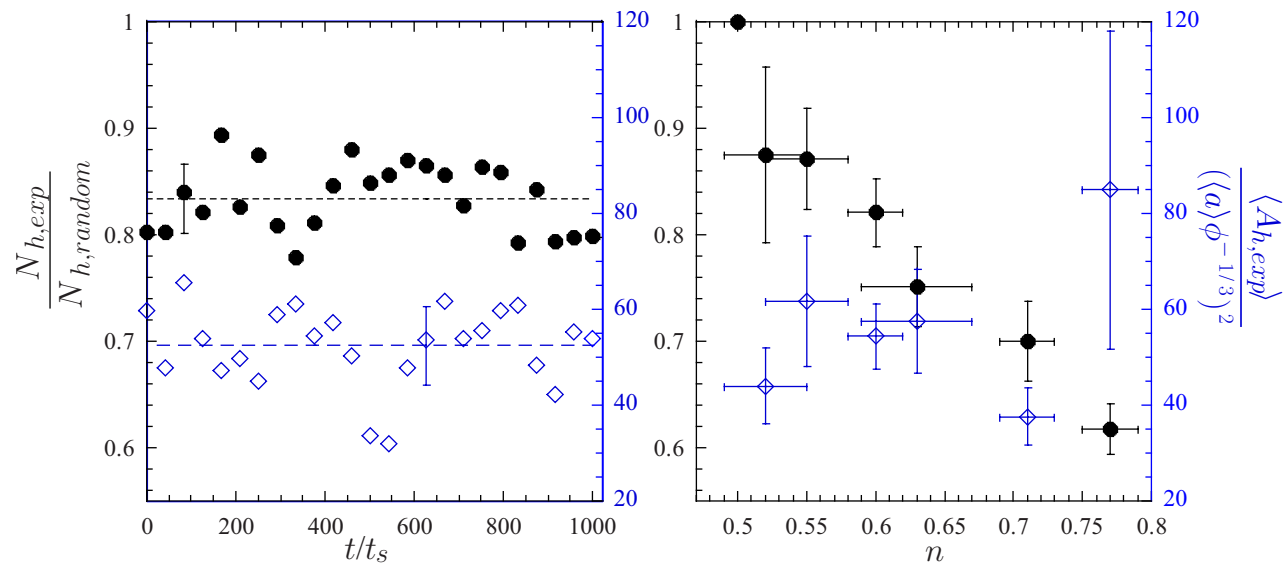

FIG. 5. (Color online) Hole number $N_{h, \exp }(\bullet)$ and hole area $A_{h, \exp }(\diamond)$ [dark gray (blue online)] vs time for particles of batch B at $\phi=0.3 \%$ (left) and vs $n$ for the different experiments (right).

variation with time is consistent with the nonevolution of the power law seen in Fig. 3. Figure 5 (right) shows the evolution of $N_{h, \text { exp }}$ and $A_{h \text {,exp }}$ with the exponent $n$ for different batches of particles at different concentrations and in different containers. Clearly, as the exponent $n$ increases, i.e., as polydispersity, volume fraction, or cell size increases, the number of holes decreases and their area increases.

In summary, the first outcome of this study is that mixing a sedimenting suspension does not lead to a random distribution of particles, as has been assumed in most of the previous studies. Image analysis of the particle positioning reveals regions devoid of particles (holes) of the order of a few $a \phi^{-1 / 3}$ surrounded by regions where particles have an excess of close neighbors. It may be that the small propeller used for mixing creates microvorticity zones from which are expelled the particles by centrifugal forces, thus leading to hole formation. The present letter raises the issue, which clearly needs to be followed up for different approaches to mixing. The second finding is that this microstructure departs from random positioning when increasing polydispersity, particle concentration, and cell sizes. There is thus a complex interaction yet to be elucidated of a fixed mixing procedure with these parameters. A third result is that this microstructure is maintained throughout the sedimentation process. It is not yet clear what the impact of the observed microstructure has on velocity fluctuations. The holes are fluctuating structures but have a size which does not evolve in time (typically $\left.6-9 a \phi^{-1 / 3}\right)$, while that of the velocity fluctuations does (from the cell size to $\approx 20 \phi^{-1 / 3}$ ). Besides the hole size is smaller than that of the velocity fluctuations. This needs further examination, e.g., by comparing with time evolutions of Poisson and non-Poisson initial states in numerical simulations.

We thank D. Saintillan and E. J. Hinch for discussions and suggestions, D. Chehata for help in collecting data,
E. Brun, M. Nicolas, and J. Vicente for help in image analysis, and Xena for inspiration.

${ }^{1}$ G. K. Batchelor, "Sedimentation in a dilute dispersion of spheres," J. Fluid Mech. 52, 245 (1972).

${ }^{2}$ R. E. Caflisch and J. H. C. Luke, "Variance in the sedimenting speed of a suspension," Phys. Fluids 28, 759 (1985).

${ }^{3}$ E. J. Hinch, in Disorder and Mixing, edited by E. Guyon, J.-P. Nadal, and Y. Pomeau (Kluwer, Dordrecht, 1988), p. 153.

${ }^{4}$ É. Guazzelli, "Evolution of particle-velocity correlations in sedimentation," Phys. Fluids 13, 1537 (2001).

${ }^{5}$ L. Bergougnoux, S. Ghicini, É. Guazzelli, and E. J. Hinch, "Spreading fronts and fluctuations in sedimentation," Phys. Fluids 15, 1875 (2003).

${ }^{6}$ D. Chehata Gómez, L. Bergougnoux, E. J. Hinch, and É. Guazzelli, "On stratification control of the velocity fluctuations in sedimentation," Phys. Fluids 19, 098102 (2007).

${ }^{7}$ P. N. Segrè, E. Herbolzheimer, and P. M. Chaikin, "Long-range correlations in sedimentation," Phys. Rev. Lett. 79, 2574 (1997).

${ }^{8}$ D. L. Koch and E. S. G. Shaqfeh, "Screening in sedimenting suspensions," J. Fluid Mech. 224, 275 (1991).

${ }^{9}$ A. J. C. Ladd, "Sedimentation of homogeneous suspensions of nonBrownian spheres," Phys. Fluids 9, 491 (1997).

${ }^{10}$ A. Levine, S. Ramaswamy, E. Frey, and R. Bruinsma, "Screened and unscreened phases in sedimenting suspensions," Phys. Rev. Lett. 81, 5944 (1998).

${ }^{11} \mathrm{P}$. Tong and B. J. Ackerson, "Analogies between colloidal sedimentation and turbulent convection at high Prandtl numbers," Phys. Rev. E 58, R6931 (1998)

${ }^{12}$ A. J. C. Ladd, "Effect of container walls on the velocity fluctuations of sedimenting spheres," Phys. Rev. Lett. 88, 048301 (2002).

${ }^{13}$ P. J. Mucha, S.-Y. Tee, D. A. Weitz, B. I. Shraiman, and M. P. Brenner, "A model for velocity fluctuations in sedimentation," J. Fluid Mech. 501, 71 (2004).

${ }^{14}$ N.-Q. Nguyen and A. J. C. Ladd, "Sedimentation of hard-sphere suspensions at low Reynolds number," J. Fluid Mech. 525, 73 (2005).

${ }^{15} \mathrm{~L}$. Talini, "Étude de la structure de suspensions non-Browniennes en sédimentation par une nouvelle technique de R.M.N.," Ph.D. Thesis, Université de Paris 6, 1998.

${ }^{16}$ X. Lei, B. J. Ackerson, and P. Tong, "Settling statistics of hard sphere particles," Phys. Rev. Lett. 86, 3300 (2001).

${ }^{17}$ H. Edelsbrunner and E. P. Mücke, "Three-dimensional alpha shapes," ACM Trans. Graphics 13, 43 (1994).

${ }^{18}$ E. J. Hinch, private communication (2008). 